\title{
Adaptive Power Allocation for Bidirectional Amplify-and-Forward Multiple-Relay Multiple-User Networks
}

\author{
Ted C.-K. Liu, Wei Xu, Xiaodai Dong, and Wu-Sheng Lu \\ Department of Electrical and Computer Engineering, University of Victoria, Victoria, BC, Canada \\ e-mail: $\{$ tcliu,wxu, xdong, wslu\}@ece.uvic.ca
}

\begin{abstract}
Owing to its spectral efficiency, bidirectional relaying is a promising candidate for information exchange in multiple-user cooperative networks. When the network is limited by resource constraints, amplify-and-forward (AaF) relay protocol is often the choice due to its simplicity and ease of use. Power allocation for AaF protocol has being extensively studied in unidirectional relay networks but how it can be implemented in two-way multiple-relay multiple-user networks has yet to be addressed. In this paper, we consider the adaptive power allocation in bidirectional AaF multiple-relay multipleuser networks. We show that when the multiple-user interference can be removed by a robust channel assignment algorithm, power allocation by maximizing the instantaneous sum rate or minimizing the symbol error rate can be suitably casted as a geometric programming (GP) problem. Simulation results show adaptive power allocation by GP outperforms that of equal power allocation scheme particularly when there is a single serving relay, and the gain can be as substantial when there are multiple serving relays.
\end{abstract}

\section{INTRODUCTION}

Cooperative communication is a new paradigm in communication theory which is envisioned to bring forth significant improvements for both WiMax and LTE-advanced mobile systems [1]. By relaying the same message over multiple independent relay channels, the diversity order of the end users can be increased to combat fading without any significant increase in the cost and size of the mobile unit [2], [3]. Although unidirectional relay networks, i.e., one-way relaying, has been extensively studied with both decode-and-forward [4] and amplify-and-forward (AaF) [4]-[7] protocols, it is spectrally inefficient when relaying messages in between the users [8]. The groundbreaking work by Shannon on the two-way relaying channel, i.e., bidirectional relaying, in [9] bypasses this problem and ensures that the two-way communication between two users can enjoy improved spectral efficiency compared with the traditional one-way relaying [8]. Despite of the many proposed protocols for relaying, resource allocation such as power assignment is one of the most crucial aspect of the cooperative communication due to the stringent resource limitation and the unpredictable nature of the wireless medium.

Being the simplest and easiest form of relaying, power allocation for AaF protocol has been extensively studied in unidirectional relay networks [4], [6], [7], [10], [11]. Power allocation based on both signal-to-noise ratio (SNR) maximization and outage probability minimization is considered in
[10], and that for symbol error rate (SER) minimization is considered [4] for a single-relay network under Rayleigh fading. In [6], optimal power allocation based on outage minimization subject to total power constraint is studied for multiplerelay networks, where relaying is performed by orthogonal relay channels. Zhao et. al. [7] compares between optimal power allocation by maximizing the instantaneous mutual information and selecting a relay which provides the maximum end-to-end SNR in a multiple-relay system. In multiple-user setting with time-division multiple access (TDMA), Phan et. al. [11] allocates power in single serving relay networks according to several quality-of-service criteria, and jointly considers the admission control problem. For bidirectional AaF relaying, power allocation has been considered in [12][15]. In [12], power allocation aims at maximizing the average sum rate in single-relay networks. In a similar setting, [13] further considers allocation by minimizing the outage probability in Rayleigh fading channels. When there are multiple relays, power allocation according to design rules such as maximizing the instantaneous sum rate or minimizing the outage probability are studied in [14]. In multiple-user single AaF relay networks with either TDMA or frequency-division multiple access (FDMA), [15] considered power allocation by maximizing the upper bound to the instantaneous sum rate.

In this paper, we consider the adaptive power allocation in bidirectional AaF multiple-relay multiple-user networks. Although the sum of harmonic mean functions from multiplerelay multiple-user networks is often a non-linear non-convex function, we show that when the multiple-user interference (MUI) can be removed by a robust channel assignment algorithm such as the orthogonal frequency-division multiple access (OFDMA), power allocation by maximizing the instantaneous sum rate or minimizing the asymptotic symbolerror rate (SER) subject to total power and individual power constraints can be suitably casted as a geometric programming (GP) problem [16], [17] which can be efficiently solved by the convex optimization algorithm [18]. Simulation results show adaptive power allocation by GP outperforms that of equal power allocation scheme particularly when there is a single serving relay, and the gain can be as substantial when there are multiple serving relays in the network.

The rest of this paper is as follows: Section (Sec.) II describes the system model, Sec. III outlines the power al- 
location problem by maximizing the instantaneous sum rate, Sec. IV outlines the power allocation problem by minimizing the asymptotic SER, Sec. V evaluates our adaptive power allocation schemes and compares to the equal power allocation scheme. Finally, concluding remarks are provided in Sec. VI.

\section{SySTEM MODEL}

The system model for the $k$ th pair of users in bidirectional multiple-relay multiple-user network is shown in Fig. 1. We consider $K$ pairs of users for a total of $2 K$ users. Each pair of users communicates with a fixed $J$ number of relays for $j=1,2, \ldots, J$ in half-duplex mode. Each user within a pair of users communicates in a two-phase transmission. We assume all active relays ${ }^{1}$ to transmit in orthogonal time slots in a predefined order according to certain rules, and we further assume that there is no MUI in between all of the pairs of users $^{2}$. For example, Fig. 2 illustrates the channel assignment

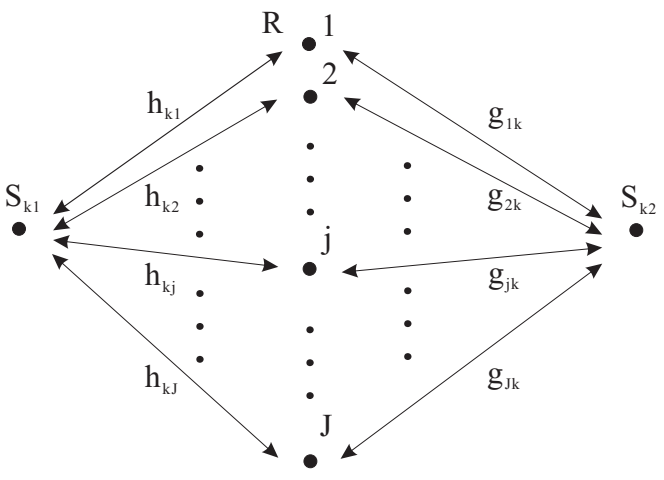

Fig. 1: System model of the $k$ th pair of users for the bidirectional AaF multiple-relay multiple-user network. A pair of users consists of 2 sources $S_{k i}, i=1,2, J$ relays, and there are a total of $K$ pairs of users in the network.

scheme employing the OFDMA for $J$ relays and $2 K$ multiple users. In the first phase, both sources from the $k$ th pair of users broadcast to all relays simultaneously. The received signal at the $j$ th relay can be written as

$$
y_{k j}=\sqrt{P_{S_{k 1}}} h_{k j} x_{k 1}+\sqrt{P_{S_{k 2}}} g_{j k} x_{k 2}+n_{k j},
$$

where $h_{k j}$ and $g_{j k}$ are the fading channel gains between $S_{k 1}$ and the $j$ th relay and between $S_{k 2}$ and the $j$ th relay, respectively, $P_{S_{k i}}, i \in\{1,2\}$ are the transmitter source powers, $x_{k i}, i \in\{1,2\}$ are the transmit source symbol normalized to unit energy, i.e., $\mathbb{E}\left\{\left|x_{k i}\right|^{2}\right\}=1$, and $n_{k j} \sim \mathcal{C N}\left(0, \sigma_{n_{k j}}^{2}\right)$ is the additive complex symmetric zero-mean white Gaussian noise (CSZWGN) with variance $\sigma_{n_{k j}}^{2}=N_{k j}$. The channels are assumed to be independently distributed Rayleigh flat fading

\footnotetext{
${ }^{1}$ By active relays, we meant the set of relays that are involved in forwarding the information to the destination which may not necessary be every relay within the entire network, but can be either a subset of relays or a single relay depending on the relaying protocol.

${ }^{2}$ This assumption can be realized by developing a robust channel assignment algorithm such as the OFDMA, or hybrid TDMA and FDMA schemes which can put multiple users into orthogonal channels to mitigate MUI. The development of such algorithms is beyond the scope of this paper.
}

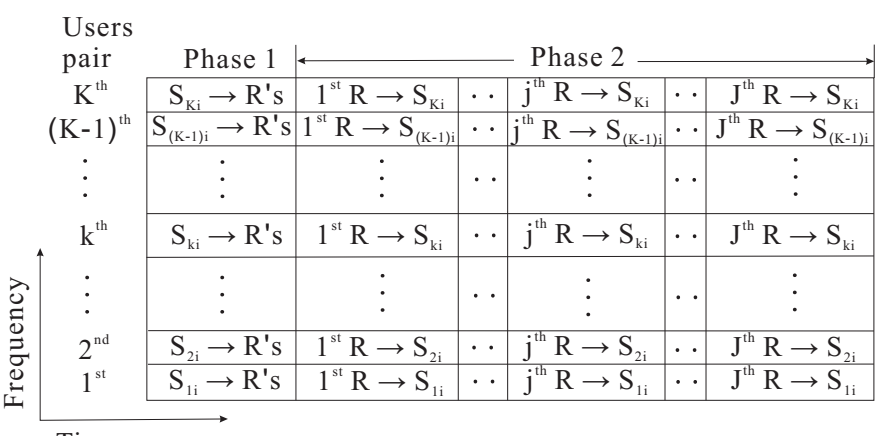

Time

Fig. 2: The OFDMA channel assignment scheme for the bidirectional AaF multiple-relay multiple-user network employing $J$ relays.

with variances modeled according to uniform path loss, with a path loss exponent $\alpha$. Let variables $d_{k j 1}$ and $d_{j k 2}$ denote the distance between the pair of users to the $j$ th relay node, then we have $\sigma_{h_{k j}}^{2}=\bar{\gamma}_{h_{k j}}=d_{k j 1}^{-\alpha}$ and $\sigma_{g_{j k}}^{2}=\bar{\gamma}_{g_{j k}}=d_{j k 2}^{-\alpha}$ for channels $h_{k j}$ and $g_{j k}$, respectively. We assume the channels to remain static during the entire phase of the multiple-user relay communication.

The active relay then scales the received signal energy according to the instantaneous power scaling (IPS) rule and forwards the message to both sources in the second phase in accordance to the AaF protocol [2]. The received signal at the two sources of the $k$ th pair of users from the $j$ th relay can now be written as

$$
\begin{aligned}
y_{S_{k 1}} & =\sqrt{P_{k j} P_{S_{k 2}}} h_{k j} g_{j k} \beta_{k j} x_{k 2}+\sqrt{P_{k j} P_{S_{k 1}}} h_{k j}^{2} \beta_{k j} x_{k 1}+ \\
& \sqrt{P_{k j}} h_{k j} \beta_{k j} n_{k j}+n_{S_{k 1}}, \\
y_{S_{k 2}} & =\sqrt{P_{k j} P_{S_{k 1}}} g_{j k} h_{k j} \beta_{k j} x_{k 1}+\sqrt{P_{k j} P_{S_{k 2}}} g_{j k}^{2} \beta_{k j} x_{k 2}+ \\
& \sqrt{P_{k j}} g_{j k} \beta_{k j} n_{k j}+n_{S_{k 2}},
\end{aligned}
$$

where $P_{k j}$ is defined as the $j$ th relay power for the $k$ th pair of users, $n_{S_{k i}} \sim \mathcal{C N}\left(0, \sigma_{n_{S_{k i}}}^{2}\right), i \in\{1,2\}$ is the CSZWGN at the source with variance $\sigma_{n_{S_{k i}}}^{2}=N_{S_{k i}}$. The second term in (2) is the self-interference which can be removed completely since the source knows the data sent. For IPS, the square of the scaling factor is $\beta_{k j}^{2}=\frac{1}{P_{S_{k 1}}\left|h_{k j}\right|^{2}+P_{S_{k 2}}\left|g_{j k}\right|^{2}+N_{k j}}$.

We now perform the maximum ratio combining (MRC) scheme [4], [6], [7] at the pair of users as follows

$$
\begin{aligned}
z_{S_{k 1}} & =\sum_{j=1}^{J} \frac{\sqrt{P_{k j} P_{S_{k 2}}} h_{k j}^{*} g_{j k}^{*} \beta_{k j}}{\left(P_{k j}\left|h_{k j}\right|^{2} \beta_{k j}^{2}+1\right) N_{0}} y_{S_{k 1}}, \\
z_{S_{k 2}} & =\sum_{j=1}^{J} \frac{\sqrt{P_{k j} P_{S_{k 1}}} h_{k j}^{*} g_{j k}^{*} \beta_{k j}}{\left(P_{k j}\left|g_{j k}\right|^{2} \beta_{k j}^{2}+1\right) N_{0}} y_{S_{k 2}},
\end{aligned}
$$

where $(\cdot)^{*}$ denotes the complex conjugate operation, and we have assumed that the noise components are independently and identically distributed (i.i.d.) CSZWGN with common variance $N_{0}$, i.e., $N_{k j}=N_{S_{k i}}=N_{0}$. For the MRC in (3), we have assumed that both sources can obtain perfect channel state 
information (CSI) and the CSI from the opposite source can be acquired through an error-free feedback channel. Substituting $\beta_{k j}$ into (3), the end-to-end signal-to-noise ratio (SNR) then becomes

$$
\gamma_{k 1}=\sum_{j=1}^{J} \gamma_{k j 1}, \text { and } \gamma_{k 2}=\sum_{j=1}^{J} \gamma_{k j 2}
$$

where $\gamma_{k j 1}=\frac{P_{k j} \gamma_{h_{k j}} P_{S_{k 2}} \gamma_{g_{j k}}}{\left(P_{k j}+P_{S_{k 1}}\right) \gamma_{h_{k j}}+P_{S_{k 2}} \gamma_{g_{j k}}+1}$ and $\gamma_{k j 2}=$ $\frac{P_{k j} P_{S_{k 1}} \gamma_{h_{k j}} \gamma_{g_{j k}}}{P_{S_{k 1}} \gamma_{h_{k j}}+\left(P_{k j}+P_{S_{k 2}}\right) \gamma_{g_{j k}}+1}$ are the per relay end-to-end SNR. The variables $\gamma_{k j i}, i=1,2$, can be approximated as a harmonic mean of two random variables (r.v.'s) at high SNR [4], [5], i.e., $\frac{x_{1} x_{2}}{x_{1}+x_{2}}$ for r.v.'s $x_{1}$ and $x_{2}$. For the SNR terms in (4), $\gamma_{h_{k j}}=\frac{\left|h_{k j}\right|^{2}}{N_{0}}$ is the instantaneous SNR between the source $S_{k 1}$ and the $j$ th relay, and $\gamma_{g_{j k}}=\frac{\left|g_{j k}\right|^{2}}{N_{0}}$ is the instantaneous SNR between the $j$ th relay and source $S_{k 2}$.

\section{Power Allocation By Maximizing InStANTANEOUS SUM RATE}

Assuming all relaying are performed in orthogonal time slots, the optimization problem to maximize the instantaneous sum rate in the multiple-user network can be formulated as ${ }^{3}$

$$
\begin{aligned}
& \max _{\left\{P_{S_{k 1}}, P_{S_{k 2}}, P_{k j}\right\}} \sum_{k=1}^{K} A_{k} \times \\
& \left\{\log _{2}\left(1+\sum_{j=1}^{J} \gamma_{k j 1}\right)+\log _{2}\left(1+\sum_{j=1}^{J} \gamma_{k j 2}\right)\right\}, \\
& \text { s.t. } \sum_{k=1}^{K} P_{S_{k 1}}+\sum_{k=1}^{K} P_{S_{k 2}}+\sum_{k=1}^{K} \sum_{j=1}^{J} P_{k j} \leq P_{T O T}, \\
& 0 \leq P_{S_{k i}} \leq P_{S_{k i}}^{\mathrm{MAx}}, \quad k=1, \ldots, K, i=1,2, \\
& 0 \leq P_{k j} \leq P_{k j}^{\mathrm{MAX}}, \quad k=1, \ldots, K, j=1, \ldots, J,
\end{aligned}
$$

where $P_{T O T}$ is the total power constraint of the entire multiple-user network, $A_{k}=\frac{1}{J_{k}+1}$ is the bandwidth factor, $J_{k}=\left|\mathcal{J}_{k}\right|$ is the number of active relays connecting to the $k$ th pair of users so $\mathcal{J}_{k}$ is the set of active relays connecting to the $k$ th pair of users. Hence, $A_{k}$ is a variable value that depends on the number of relays being active, i.e., $P_{k j} \neq 0, j=1, \ldots, J$, during the $k$ th pair of users transmission ${ }^{4}$. The constraints in (6)-(8) are constraints to total network power, individual pair of users power, and individual relay to pair of users power. The variables $P_{S_{k i}}^{\mathrm{MAX}}, k=1, \ldots, K, i=1,2$, and $P_{k j}^{\mathrm{MAX}}$, $k=1, \ldots, K, j=1, \ldots, J$, are upper bounds on user powers and individual relay powers, respectively, which can be used to maximize the network lifetime if needed.

\footnotetext{
${ }^{3}$ We can also consider the weighted sum rate by the use of pre-log weights $w_{k i}, i=1,2$ to represent the priority assignments to each source [14]. For simplicity of the problem herein, we let $w_{k i}=1, i=1,2, \forall k$.

${ }^{4}$ For simplicity of this paper, we assume $A_{k}=A=\frac{1}{J+1}, \forall k$ such that all pairs of users employ the same number of relays $J$ for relay communication.
}

\section{A. Single-relay Two-way System}

For this paper, we may simplify (5)-(8) by assuming that there exists a single relay that is used to serve multiple pairs of users then the objective function can be expressed as

$$
\begin{aligned}
& \sum_{k=1}^{K} \frac{1}{2}\left\{\log _{2}\left(1+\gamma_{k 1}\right)+\log _{2}\left(1+\gamma_{k 2}\right)\right\} \\
& \geq \sum_{k=1}^{K} \frac{1}{2} \log _{2}\left(\gamma_{k 1} \gamma_{k 2}\right)=-\sum_{k=1}^{K} \frac{1}{2} \log _{2}\left(\gamma_{k 1}^{-1} \gamma_{k 2}^{-1}\right),
\end{aligned}
$$

where $\gamma_{k 1}=\frac{P_{k 1} \gamma_{h_{k 1}} P_{S_{k 2}} \gamma_{g_{1 k}}}{\left(P_{k 1}+P_{S_{k 1}}\right) \gamma_{h_{k 1}}+P_{S_{k 2}} \gamma_{g_{1 k}}+1}, \quad \gamma_{k 2}=$ $\frac{P_{k 1} P_{S_{k 1}} \gamma_{h_{k 1}} \gamma_{g_{1 k}}}{P_{S_{k 1}} \gamma_{h_{k 1}}+\left(P_{k 1}+P_{S_{k 2}}\right) \gamma_{g_{1 k}}+1}$. The above objective function can be turned to an equivalent minimization problem that can be solved by geometric programming (GP) [16], [17] with an efficient convex optimization algorithm such as the CVX [18]. Transforming the summation into the $\log _{2}(\cdot)$ argument as a product in (9), the equivalent problem in (5)-(8) for single-relay system can now be stated as

$$
\begin{gathered}
\min _{\left\{P_{S_{k 1}}, P_{S_{k 2}}, P_{k 1}\right\}} \prod_{k=1}^{K} z_{k 1} z_{k 2}, \\
\text { s.t. } \frac{\left(P_{k 1}+P_{S_{k 1}}\right) \gamma_{h_{k 1}}+P_{S_{k 2}} \gamma_{g_{1 k}}+1}{P_{k 1} \gamma_{h_{k 1}} P_{S_{k 2}} \gamma_{g_{1 k}}} \leq z_{k 1}, \\
\frac{P_{S_{k 1}} \gamma_{h_{k 1}}+\left(P_{k 1}+P_{S_{k 2}}\right) \gamma_{g_{1 k}}+1}{P_{k 1} P_{S_{k 1}} \gamma_{h_{k 1}} \gamma_{g_{1 k}}} \leq z_{k 2}, \\
\sum_{k=1}^{K} P_{S_{k 1}}+\sum_{k=1}^{K} P_{S_{k 2}}+\sum_{k=1}^{K} P_{k 1} \leq P_{T O T}, \\
0 \leq P_{S_{k i}} \leq P_{S_{k i}}^{\mathrm{MAx}}, \quad k=1, \ldots, K, i=1,2, \\
0 \leq P_{k 1} \leq P_{k 1}^{\mathrm{MAX}}, \quad k=1, \ldots, K, \\
z_{k 1} \geq 0, z_{k 2} \geq 0, \quad k=1, \ldots, K .
\end{gathered}
$$

\section{B. Multiple-relay Two-way System}

For simplicity, we assume there is no relay selection so the problem is more complicated than the single-relay case. Motivated by [14], our main idea is to solve for the simpler suboptimal power allocation problem by maximizing the lower bound to the high SNR sum capacity approximation. The high SNR sum capacity lower bound can be written as

$$
\begin{aligned}
& \sum_{k=1}^{K} \frac{1}{J+1} \log _{2}\left(\sum_{j=1}^{J} \gamma_{k j 1} \gamma_{k j 2}\right) \\
& \geq \sum_{k=1}^{K} \frac{1}{J(J+1)} \sum_{j=1}^{J} \log _{2}\left(\gamma_{k j 1} \gamma_{k j 2}\right)+C \\
& =-\sum_{k=1}^{K} \sum_{j=1}^{J} \log _{2}\left(\gamma_{k j 1}^{-\frac{1}{J(J+1)}} \gamma_{k j 2}^{-\frac{1}{J(J+1)}}\right)+C,
\end{aligned}
$$

where $C=-\sum_{k} \frac{1}{J+1} \log _{2}\left(\frac{1}{J}\right)$ is a constant term which can be removed in the optimization. The second line in (17) follows from Jensen's inequality. So, the final problem is equivalent to maximizing the lower bound to the original 
problem, c.f., (5), without the high SNR approximation.

Now, transforming the summations into the argument of $\log _{2}(\cdot)$ as products, the power allocation optimization problem can be formulated into a GP problem as

$$
\begin{gathered}
\min _{\left\{P_{S_{k 1}}, P_{S_{k 2}}, P_{k j}\right\}} \prod_{k=1}^{K} \prod_{j=1}^{J} z_{k j 1}^{\frac{1}{J(J+1)}} z_{k j 2}^{\frac{1}{J(J+1)}}, \\
\text { s.t. } \frac{\left(P_{k j}+P_{S_{k 1}}\right) \gamma_{h_{k j}}+P_{S_{k 2}} \gamma_{g_{j k}}+1}{P_{k j} \gamma_{h_{k j}} P_{S_{k 2}} \gamma_{g_{j k}}} \leq z_{k j 1}, \\
\\
\frac{P_{S_{k 1}} \gamma_{h_{k j}}+\left(P_{k j}+P_{S_{k 2}}\right) \gamma_{g_{j k}}+1}{P_{k j} P_{S_{k 1}} \gamma_{h_{k j}} \gamma_{g_{j k}}} \leq z_{k j 2}, \\
\\
\sum_{k=1}^{K} P_{S_{k 1}}+\sum_{k=1}^{K} P_{S_{k 2}}+\sum_{k=1}^{K} \sum_{j=1}^{J} P_{k j} \leq P_{T O T}, \\
0 \leq P_{S_{k i}} \leq P_{S_{k i}}^{\mathrm{MAX}}, \quad k=1, \ldots, K, i=1,2, \\
0 \leq P_{k j} \leq P_{k j}^{\mathrm{MAX}}, \quad k=1, \ldots, K, j=1, \ldots, J, \\
z_{k j 1} \geq 0, z_{k j 2} \geq 0, \quad k=1, \ldots, K, j=1, \ldots, J .
\end{gathered}
$$

For the GP in (18)-(24), care must be made such that each $z_{k j i}, i=1,2$ remains as a single auxiliary variable of posynomials [16]. This can be realized by vectorization and imposing additional equality constraints on the individual source powers.

\section{Power Allocation by Minimizing SER}

We also consider the case of power allocation by minimizing the average system SER subject to both total and individual power constraints. However, minimizing the system SER is not easy due to the non-convexity of the primal problem. In order to make the power allocation problem more tractable, we design the power allocation scheme by minimizing the lower bound of the system SER. We first derive the individual user SER of the $k$ th pair of users under $M$-ary phase shifted keying (PSK) modulation with the asymptotic SNR assumption. Then, transforming the single user asymptotic SER expression into the form of power allocation by minimizing the system SER in multiple-user networks. We would like to stress that our power allocation strategy can be easily generalized to other linear digital modulations.

\section{A. Asymptotic SER of Single-pair of Users}

The SER of the $i$ th user in the $k$ th pair of users can be expressed as [19]

$$
P_{s e_{k i}}=\frac{1}{\pi} \int_{0}^{(M-1) \pi / M} \prod_{j=1}^{J} \mathcal{M}_{\gamma_{k j i}}\left(\frac{g_{\mathrm{PSK}}}{\sin ^{2} \theta}\right) \mathrm{d} \theta
$$

where $g_{\mathrm{PSK}}=\sin ^{2}(\pi / M)$, and $\mathcal{M}_{\gamma_{k j i}}(\cdot)$ is the moment generating function (MGF) of the $j$ th relay to the $i$ th user in the $k$ th pair of users. To approximate (25) in an asymptotic manner, we first rewrite the $j$ th relay end-to-end SNR for the 1st user for high SNR as follows

$$
\gamma_{k j 1} \leq \frac{P_{k j} \gamma_{h_{k j}}\left(\frac{P_{S_{k 2}}}{P_{k j}+P_{S_{k 1}}}\right) \gamma_{g_{j k}}}{\gamma_{h_{k j}}+\left(\frac{P_{S_{k 2}}}{P_{k j}+P_{S_{k 1}}}\right) \gamma_{g_{j k}}}=\frac{P_{k j} \gamma_{h_{k j}} \alpha_{k j 1} \gamma_{g_{j k}}}{\gamma_{h_{k j}}+\alpha_{k j 1} \gamma_{g_{j k}}}
$$

For Rayleigh fading channels, $\gamma_{h_{k j}}$ and $\alpha_{k j 1} \gamma_{g_{j k}}$ in (26) are exponentially distributed r.v.'s with means $\beta_{k j 1}=\bar{\gamma}_{h_{k j}}^{-1}$ and $\beta_{k j 2}=\alpha_{k j 1}^{-1} \bar{\gamma}_{g_{j k}}^{-1}$. Now, we briefly recall a useful result in [4, Theorem 4] as follows.

Theorem 1: Let $X_{1}$ and $X_{2}$ be two independent exponential r.v.'s with parameters $\beta_{1}$ and $\beta_{2}$ respectively. Then, the MGF of $Z=\frac{X_{1} X_{2}}{X_{1}+X_{2}}$ is

$$
\begin{gathered}
\mathcal{M}_{Z}(s)=\frac{\left(\beta_{1}-\beta_{2}\right)^{2}+\left(\beta_{1}+\beta_{2}\right) s}{\Delta^{2}}+\frac{2 \beta_{1} \beta_{2} s}{\Delta^{3}} \times \\
\ln \frac{\left(\beta_{1}+\beta_{2}+s+\Delta\right)^{2}}{4 \beta_{1} \beta_{2}},
\end{gathered}
$$

for any $s>0$ in which $\Delta=$ $\sqrt{\left(\beta_{1}-\beta_{2}\right)^{2}+2\left(\beta_{1}+\beta_{2}\right) s+s^{2}}$. Furthermore, if $\beta_{1}$ and $\beta_{2}$ go to zero, then the MGF of $Z$ can be approximated as $\mathcal{M}_{Z}(s) \approx \frac{\beta_{1}+\beta_{2}}{s}$.

By invoking the asymptotic property of Theorem 1 together with the linearity property of the MGF, the SER of the 1st user in the $k$ th pair of users becomes

$$
\begin{aligned}
P_{s e_{k 1}} & \geq \tilde{P}_{s e_{k 1}} \\
& =\frac{1}{\pi} \int_{0}^{(M-1) \pi / M} \frac{\sin ^{2 J} \theta}{\sin ^{2 J}(\pi / M)} \mathrm{d} \theta \prod_{j=1}^{J} \frac{\beta_{k j 1}+\beta_{k j 2}}{P_{k j}} \\
& =K^{\prime} \prod_{j=1}^{J}\left(\frac{P_{S_{k 2}} \bar{\gamma}_{g_{j k}}+\left(P_{k j}+P_{S_{k 1}}\right) \bar{\gamma}_{h_{k j}}}{P_{k j} P_{S_{k 2}} \bar{\gamma}_{h_{k j}} \bar{\gamma}_{g_{j k}}}\right)
\end{aligned}
$$

With an interchange of variables on the exponential means for the 2nd user, the average SER of the $k$ th pair of users can be lower bounded as $P_{s e_{k}} \geq \tilde{P}_{s e_{k}}=\frac{1}{2}\left(\tilde{P}_{s e_{k 1}}+\tilde{P}_{s e_{k 2}}\right)$.

\section{B. Multi-pair Two-way System}

In a system with multiple pairs of users, we allocate power based on minimizing the average SER of the entire network, i.e., $P_{\text {se тот }}=\frac{1}{K} \sum_{k=1}^{K} P_{s e_{k}}$, subject to total and individual power constraints. Due to the nonconvexity of the primal problem, we minimize the lower bound instead, i.e.,

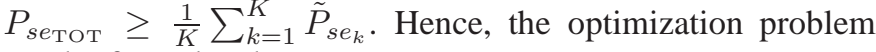
can be formulated as

$$
\begin{gathered}
\min _{\left\{P_{S_{k 1}}, P_{S_{k 2}}, P_{k j}\right\}} \sum_{k=1}^{K}\left(\prod_{j=1}^{J} t_{k j 1}+\prod_{j=1}^{J} t_{k j 2}\right), \\
\text { s.t. } \frac{\left(P_{k j}+P_{S_{k 1}}\right) \bar{\gamma}_{h_{k j}}+P_{S_{k 2}} \bar{\gamma}_{g_{j k}}}{P_{k j} P_{S_{k 2}} \bar{\gamma}_{h_{k j}} \bar{\gamma}_{g_{j k}}} \leq t_{k j 1}, \\
\frac{P_{S_{k 1}} \bar{\gamma}_{h_{k j}}+\left(P_{k j}+P_{S_{k 2}}\right) \bar{\gamma}_{g_{j k}}}{P_{k j} P_{S_{k 1}} \bar{\gamma}_{h_{k j}} \bar{\gamma}_{g_{j k}} \leq t_{k j 2},} \\
\sum_{k=1}^{K} P_{S_{k 1}}+\sum_{k=1}^{K} P_{S_{k 2}}+\sum_{k=1}^{K} \sum_{j=1}^{J} P_{k j} \leq P_{T O T},
\end{gathered}
$$




$$
\begin{aligned}
& 0 \leq P_{S_{k i}} \leq P_{S_{k i}}^{\mathrm{MAX}}, \quad k=1, \ldots, K, i=1,2, \\
& 0 \leq P_{k j} \leq P_{k j}^{\mathrm{MAX}}, \quad j=1, \ldots, J, k=1, \ldots, K, \\
& t_{k j 1} \geq 0, t_{k j 2} \geq 0, \quad j=1, \ldots, J, k=1, \ldots, K .
\end{aligned}
$$

The optimization problem above is in the form of sum of products of posynomials, which can be solved efficiently with GP. Please note that care must be observed such that $t_{k j i}, i=$ 1,2 remains as a single auxiliary variable of posynomials. The optimization problem in (29)-(35) can be easily casted for a single pair of users network by setting $K=1$.

\section{Simulation Results}

We conduct simulation with the CVX [18] optimization toolbox to verify the proposed adaptive power (Apt. Pwr.) allocation scheme while comparing to the equal power (Eq. Pwr.) allocation scheme in bidirectional AaF multiple-user network. The simulation setup is as follows. We assume independent Rayleigh fading with path loss exponent $\alpha=4$ on all of the multiple-user channels ${ }^{5}$. The distance between the two users is normalized to unity, i.e., $d_{k j 1}+d_{j k 2}=1$ for $k=1, \ldots, K$. Similar to [14], we assume that all relays are located in a line through the two pair of users to minimize the path loss, and for simplicity $d_{k j 1} \sim \mathcal{U}(0,1)$, where $\mathcal{U}(a, b)$ denotes a uniform distribution taking on the value in between $a$ and $b$, and $d_{k j 2}=1-d_{k j 1}$ for $k=1, \ldots, K$. We further assume that the entire system has a common noise variance of unity, i.e., $N_{0}=1$, and we use uncoded 4-ary PSK modulation for simplicity. The results shown are averaged over 1,000 independent trials, and 100 bit error counts.

\section{A. Power Allocation by Sum Rate Maximization}

Fig. 3 plots the average sum rate versus number of pairs of users $K$ with a single serving relay, i.e., $J=1$, as $\mathrm{SNR}^{6}$ varies from 10 to $20 \mathrm{~dB}$. Obviously, the adaptive power allocation using GP is more superior since it can attain to higher average sum rate than the equal power allocation at all SNR settings. The average sum rate increases as SNR and $K$ increase which is as expected from (5).

Fig. 4 plots the average sum rate versus total system SNR with a single serving relay, i.e., $J=1$, as $K$ takes on discrete values of 1,5 , and 11 . When there are a pair of users sharing resources, power allocation by adaptive method clearly outperforms that of the equal allocation. However, as $K$ increases the gain in optimization diminishes since less power is distributed to a particular user. The sum rate increases as $K$ increases which conforms to (5).

We plot Fig. 5 to validate our adaptive power allocation scheme over equal allocation scheme in a multiple-relay system. We assume a $K=2$, i.e., 4 users, system with $J=\{1,3,6,9\}$. As shown, adaptive allocation scheme outperforms the equal allocation scheme. However, both schemes suffer from a diminishing sum rate as $J$ increases since the rate decreases as $J$ increases, c.f., (5). In the case when the

\footnotetext{
${ }^{5}$ Alternatively, we may also assume i.i.d. Rayleigh fading channels but the improvements from our power allocation scheme would remain identical.

${ }^{6}$ We define the SNR as the total system SNR, i.e., $P_{T O T} / N_{0}$.
}

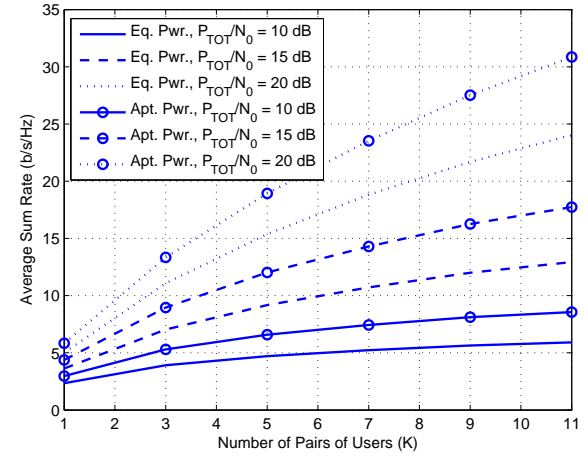

Fig. 3: Average sum rate versus number of pairs of users $K$ with a single serving relay with total system SNR $P_{T O T} / N_{0}=$ $\{10,15,20\} \mathrm{dB}$ in bidirectional AaF multiple-user network.

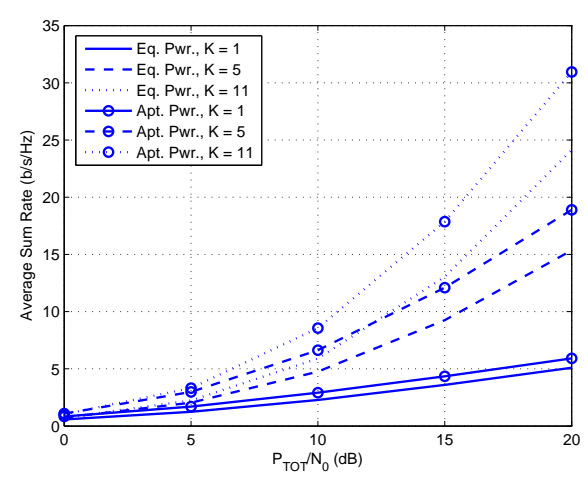

Fig. 4: Average sum rate versus total system SNR with a single serving relay with a varying $K=\{1,5,11\}$ in bidirectional AaF multiple-user networks.

CSI are known a priori at the relays, we can select the best relay or a subset of good CSI relays to forward the message thus overcoming the diminishing sum rate problem. The issue of relay selection is beyond the scope of this paper.

\section{B. Power Allocation by Minimizing SER}

We confirm the robustness of our SER minimization algorithm first in a single-pair network, i.e., $K=1$ for 2 users. Fig. 6 plots the average SER as the total system SNR varies, and we have included (27) from Theorem 1 to evaluate the SER lower bounds. Clearly, our scheme outperforms that of equal power allocation while both match fairly well to the MGF lower bounds. The negligible differences in between (27) and simulations for the case of power optimization are namely due to numerical round off errors.

Fig. 7 plots the average system SER versus total system SNR in bidirectional multiple-user networks with $K=2$, i.e., 4 users, as $J=\{1,3,5\}$, with the SER lower bounds from Theorem 1. Similar to the single pair of users case, our adaptive power allocation scheme significantly outperforms that of the equal power allocation. Furthermore, the simulation 


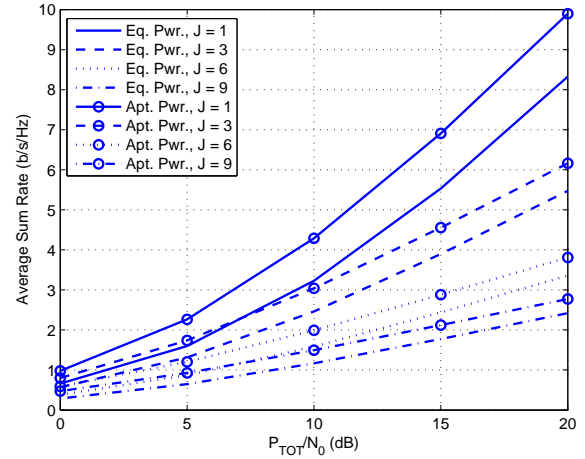

Fig. 5: Average sum rate versus total system SNR in a system with $K=2$ as $J$ takes on values $J=\{1,3,6,9\}$ in bidirectional AaF multiple-user networks.

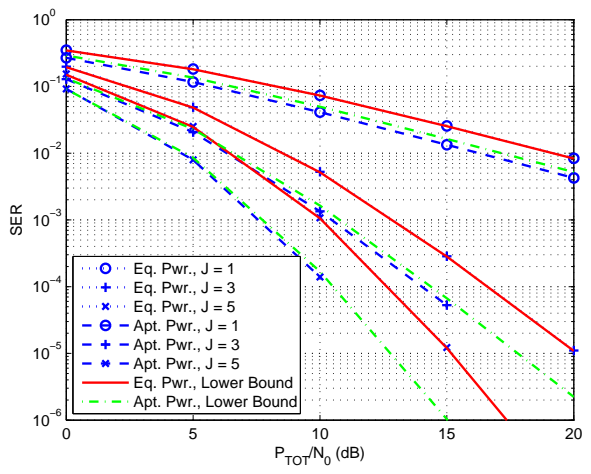

Fig. 6: Average SER versus total system SNR in a system with $K=1$ as $J=\{1,3,5\}$.

results for the optimization cases match well with the SER lower bounds.

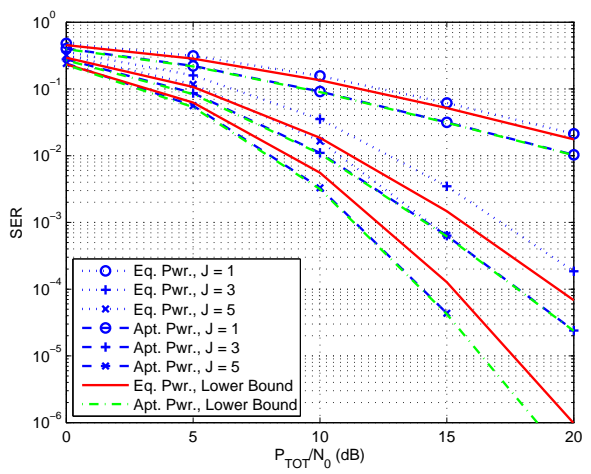

Fig. 7: Average system SER versus total system SNR with $K=2$ as $J=\{1,3,5\}$.

\section{CONCLUSION}

In this paper, we have considered the adaptive power allocation in bidirectional $\mathrm{AaF}$ multiple-relay multiple-user networks. We have shown that with the OFDMA channel assignment scheme, power allocation by maximizing the instantaneous sum rate or minimizing the SER subject to total power and individual power constraints can be suitably casted as a GP problem. Simulation results have shown that adaptive power allocation by GP outperforms that of the equal power allocation scheme.

\section{ACKNOWLEDGMENT}

This work was supported by the Natural Sciences and Engineering Research Council (NSERC) of Canada.

\section{REFERENCES}

[1] Y. Yang, H. Hu, J. Xu, and G. Mao, "Relay technologies for wimax and lte-advanced mobile systems," IEEE Commun. Mag., vol. 47, no. 10, pp. 100-105, Oct. 2009.

[2] J. Laneman, D. Tse, and G. Wornell, "Cooperative diversity in wireless networks: Efficient protocols and outage behavior," IEEE Trans. Inf. Theory, vol. 50, no. 12, pp. 3062-3080, Dec. 2004.

[3] A. Sendonaris, E. Erkip, and B. Aazhang, "User cooperation diversity. Part I. System description," IEEE Trans. Commun., vol. 51, no. 11, pp. 1927-1938, Nov. 2003.

[4] W. Su, A. Sadek, and K. Ray Liu, "Cooperative communication protocols in wireless networks: performance analysis and optimum power allocation," Wireless Personal Communications, vol. 44, no. 2, pp. 181217, 2008.

[5] S. Ikki and M. Ahmed, "Performance analysis of cooperative diversity wireless networks over Nakagami-m fading channel," IEEE Commun. Lett., vol. 11, no. 4, pp. 334-336, Apr. 2007.

[6] K. Seddik, A. Sadek, W. Su, and K. Liu, "Outage analysis and optimal power allocation for multinode relay networks," IEEE Signal Process. Lett., vol. 14, no. 6, pp. 377-380, June 2007.

[7] Y. Zhao, R. Adve, and T. Lim, "Improving amplify-and-forward relay networks: optimal power allocation versus selection," IEEE Trans. Wireless Commun., vol. 6, no. 8, pp. 3114-3123, Aug. 2007.

[8] B. Rankov and A. Wittneben, "Spectral efficient protocols for halfduplex fading relay channels," IEEE J. Sel. Areas Commun., vol. 25, no. 2, pp. 379-389, Feb. 2007.

[9] C. Shannon, "Two-way communication channels," in Proc. 4th Berkeley Symp. Math. Stat. Probab., vol. 1, 1961, pp. 611-644.

[10] X. Deng and A. Haimovich, "Power allocation for cooperative relaying in wireless networks," IEEE Commun. Lett., vol. 9, no. 11, pp. 994-996, Nov. 2005.

[11] K. Phan, T. Le-Ngoc, S. Vorobyov, and C. Tellambura, "Power allocation in wireless multi-user relay networks," IEEE Trans. Wireless Commun., vol. 8, no. 5, pp. 2535-2545, May 2009.

[12] Y. Han, S. Ting, C. Ho, and W. Chin, "High rate two-way amplify-andforward half-duplex relaying with OSTBC," in Proc. of IEEE VTC, May 2008, pp. 2426-2430.

[13] Y. Zhang, Y. Ma, and R. Tafazolli, "Power allocation for bidirectional af relaying over rayleigh fading channels," IEEE Commun. Lett., vol. 14, no. 2, pp. 145-147, Feb. 2010.

[14] X. J. Zhang and Y. Gong, "Adaptive power allocation in two-way amplify-and-forward relay networks," in Proc. of IEEE ICC, June 2009.

[15] M. Chen and A. Yener, "Power allocation for F/TDMA multiuser twoway relay networks," IEEE Trans. Wireless Commun., vol. 9, no. 2, pp. 546-551, Feb. 2010.

[16] S. Boyd, S. Kim, L. Vandenberghe, and A. Hassibi, "A tutorial on geometric programming," Optimization and Engineering, vol. 8, no. 1, pp. $67-127,2007$.

[17] M. Chiang, C. Tan, D. Palomar, D. O'Neill, and D. Julian, "Power Control By Geometric Programming," IEEE Trans. Wireless Commun., vol. 6, no. 7, pp. 2640-2651, July 2007.

[18] M. Grant, S. Boyd, and Y. Ye, "CVX: Matlab software for disciplined convex programming (web page and software)," June 2009. [Online]. Available: http://stanford.edu/ boyd/cvx/index.html

[19] M. Simon and M. Alouini, Digital communication over fading channels: a unified approach to performance analysis. New York: John Wiley \& Sons, 2000 